\title{
Cognitive Deficits in a Genetic Mouse Model of the Most Common Biochemical Cause of Human Mental Retardation
}

\author{
Leze Zagreda, ${ }^{1}$ Jason Goodman,, ${ }^{1}$ Donald P. Druin,, ${ }^{1}$ David McDonald, ${ }^{2}$ and Adele Diamond ${ }^{1}$ \\ ${ }^{1}$ Center for Developmental Cognitive Neuroscience, Eunice Kennedy Shriver Center, Waltham, Massachusetts 02452, \\ and ${ }^{2}$ Department of Biological Sciences, Wichita State University, Wichita, Kansas 67260-0026
}

Phenylalanine hydroxylase (Pah)-deficient "PKU mice" have a mutation in the Pah gene that causes phenylketonuria (PKU) in humans. PKU produces cognitive deficits in humans if it is untreated. We report here the first evidence that the genetic mouse model of PKU ( $\left.P a h^{\text {enu2 }}\right)$ also produces cognitive impairments. PKU mice were impaired on both odor discrimination reversal and latent learning compared with heterozygote littermates and with wild-type mice of the same BTBR strain. A small container of cinnamon-scented sand was presented on the right or left, and nutmeg-scented sand was presented on the other side; left-right location varied over trials. Digging in sand of the correct scent was rewarded by finding phenylalanine-free chocolate. To prevent scent cuing, new containers were used on every trial, and both containers always contained chocolate. Digging in the incorrect choice was stopped before the chocolate was uncovered. Once criterion was reached, the other scent was rewarded. PKU mice were impaired on reversals 2,3 , and 4 . They were also impaired in latent learning. On day 1 , half the mice were allowed to explore a maze and discover the location of water. On day 2, all mice were water-deprived and were placed in the maze. Whereas pre-exposed wild-type and heterozygous mice showed evidence that they remembered the location of the water and hence could find the water faster on day 2, pre-exposed PKU mice showed no significant benefit from their pre-exposure on day 1.

Key words: PKU; phenylketonuria; mental retardation; reversal learning; latent learning; odor discrimination; mice; learning set; memory; animal models
Phenylketonuria (PKU) is an inborn error of metabolism in which phenylalanine hydroxylase $(P a h)$, the enzyme necessary for the hydroxylation of phenylalanine (Phe) to tyrosine is absent or markedly deficient (Woo et al., 1983; Lidsky et al., 1985; DiLella et al., 1986). If PKU is left untreated, widespread brain damage and severe mental retardation result (Hsia, 1966; Cowie, 1971; Tourian and Sidbury, 1978; Koch et al., 1982). Indeed, it is the most common biochemical cause of mental retardation in people.

A genetic mouse model of PKU, called the $\mathrm{Pah}^{\text {епи } 2}$ mouse, has been created by McDonald and colleagues in the BTBR mouse strain (McDonald et al., 1990; Shedlovsky et al., 1993). PKU $\left(P h^{\text {enu } 2}\right)$ mice have a mutation of the Pah gene, homologous to the $P A H$ gene that causes PKU in humans. The $P a h^{\text {enu } 2}$ mutation results in a phenotype closely resembling human PKU; mice of the BTBR strain with the $P a h^{\text {enu } 2}$ mutation have minimal or absent Pah activity, plasma levels of Phe that are 10-20 times normal $(23 \pm 1.0 \mathrm{mg} / \mathrm{dl})$, and hypopigmentation, all of which are comparable with that seen in human PKU. However, it had never been demonstrated that the mouse model has the most prominent characteristic of human PKU: cognitive impairments. We report

Received Feb. 4, 1999; revised April 6, 1999; accepted May 4, 1999.

This work was supported by National Institute of Child Health and Human Development Grants R01 HD34346 and HD35453 to A.D., and by National Science Foundation Grant EPS-9550487 and matching funds from the state of Kansas to D.M. We thank Camellia Symonowicz for taking such good care of our animals and for help in breeding them, Michelle Michalak and Gabrielle White for technical assistance, and the New England Newborn Screening Program at the University of Massachusetts Medical Center (headed by Donna Johnson) for HPLC analyses of amino acid levels. William Dube and Jason Langlois collaborated with us in pretesting the procedures for the odor discrimination and reversals tests used here.

Correspondence should be addressed to Adele Diamond, Director, Center for Developmental Cognitive Neuroscience, Eunice Kennedy Shriver Center, 200 Trapelo Road, Waltham, MA 02452.

Copyright (C) 1999 Society for Neuroscience $\quad 0270-6474 / 99 / 196175-08 \$ 05.00 / 0$ here the first evidence of cognitive impairments in the genetic mouse model of the most common biochemical cause of mental retardation: PKU.

\section{Background: development and characterization of the genetic mouse model of PKU}

Inbred male mice of the BTBR strain were treated with the germ line mutagen ethylnitrosourea (ENU) and then mated to normal BTBR females. The resulting progeny were potential carriers of mutations at the Pah locus; 150 of these progeny were inbred in two further generations and then subjected to a Phe clearance test to identify a mouse mutant deficient in Pah activity (McDonald et al., 1990). In a second round of mutational analysis, Shedlovsky et al. (1993) identified carriers of two new mutant alleles at the Pah locus by genetic noncomplementation with the first mutant allele. For each new mutant allele, a congenic inbred mutant BTBR line was established by backcrossing to the wildtype BTBR strain for $>10$ generations to separate the Pah mutation from other, loosely linked, mutations.

Among 350 potential carriers, each representing one mutagenized paternal gamete, Shedlovsky and colleagues (1993) isolated the $\mathrm{Pah}^{\text {enu } 2}$ mutation, which resulted in a phenotype with gross elevations in blood levels of Phe and only trace amounts of activity of the Pah enzyme, closely simulating human PKU. Whereas wild-type BTBR mice show Pah activity of $40 \pm 6(\Delta$ O.D. $\times 10^{3} / \mathrm{min} / \mathrm{mg}$ protein), Pah ${ }^{\text {enu } 2}$ mice show Pah activity of $0 \pm 2$. Mixing experiments demonstrated that the mutant liver extracts did not contain an inhibitor of Pah activity. Pah mRNA was measured using RNase protection assay with total liver RNA; its level is only $1 \%$ in $\mathrm{Pah}^{\text {enu } 2}$ animals. 


\begin{tabular}{|c|c|c|c|c|}
\hline & $\begin{array}{l}\text { Total } \\
\text { number }\end{array}$ & $\begin{array}{l}\text { Number } \\
\text { of males }\end{array}$ & $\begin{array}{l}\text { Number } \\
\text { of females }\end{array}$ & $\begin{array}{l}\text { Mean age } \\
\text { in months }\end{array}$ \\
\hline \multicolumn{5}{|l|}{ Odor discrimination and reversal task } \\
\hline Wild-type BTBR mice & 19 & 8 & 11 & $3.5( \pm 2.91)$ \\
\hline Heterozygous BTBR mice & 9 & 5 & 4 & $6.3( \pm 3.56)$ \\
\hline Homozygous $\mathrm{Pah}^{\text {enu } 2}$ (PKU) mice & 12 & 4 & 8 & $4.1( \pm 1.27)$ \\
\hline \multicolumn{5}{|l|}{ Latent learning } \\
\hline Wild-type BTBR mice & 30 & 14 & 16 & $5.12( \pm 2.85)$ \\
\hline Heterozygous BTBR mice & 31 & 20 & 10 & $7.42( \pm 3.59)$ \\
\hline Homozygous Pah $^{\text {enu } 2}$ (PKU) mice & 18 & 6 & 12 & $7.98( \pm 4.53)$ \\
\hline
\end{tabular}

\section{MATERIALS AND METHODS}

\section{Subjects}

The subject groups were as follows: (1) wild-type BTBR mice (whose mean plasma Phe level was $0.85 \pm 0.17 \mathrm{mg} / \mathrm{dl}$; (2) heterozygous BTBR mice (wild type crossed with $\mathrm{Pah}^{\text {enu } 2}$ (PKU); the mean plasma Phe level of the heterozygotes was $0.93 \pm 0.30 \mathrm{mg} / \mathrm{dl}$; and (3) homozygous $P a h^{\text {enu } 2}$ (PKU) mice (whose mean plasma Phe level was $18.60 \pm 2.69 \mathrm{mg} / \mathrm{dl}$ ). Plasma Phe levels were determined by HPLC analyses performed by the New England Newborn Screening Program at the University of Massachusetts Medical Center, which performs these analyses on blood samples from every child born in the New England area. The progenitor mice were obtained from The Jackson Laboratory (Bar Harbor, ME). Most heterozygotes and $\mathrm{Pah}^{\text {enu } 2}$ (PKU) mice were produced by crossing ho-

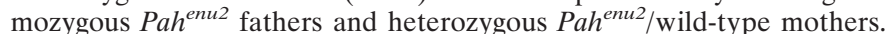
Because male $\mathrm{Pah}^{\text {enu } 2}$ (PKU) mice are not good breeders, we also set up a few matings in which both the male and female were heterozygous

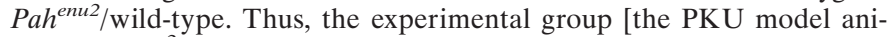
mals $\left(\mathrm{Pah}^{\text {enu2 }}\right)$ ] was compared with two control groups: their heterozygous littermates and normal animals. Mice who are heterozygous for PKU, like humans who are heterozygous for PKU, have plasma Phe levels that are almost as low as conspecifics who carry no mutation for PKU. It was important that the pregnant dams not have plasma Phe levels 10-20 times normal (as do PKU mice, or humans with PKU, on a normal diet) because that can cause in utero brain damage to the fetuses. For this reason, it was male $\mathrm{Pah}^{\text {enu } 2}$ (PKU) mice who were used for mating, never female $P_{a h} h^{\text {еnu } 2}$ (PKU) mice. Information on the ages and percentage of males and females tested on the behavior tasks is provided in Table 1. All animals were treated in accordance with the NIH Guide for the Care and Use of Animals in Research.

\section{Odor discrimination and reversal}

Two plastic bottle caps filled with scented sand were mounted on a horizontal platform, separated by a vertical barrier, and attached to a long handle. Before testing, the animals were taught to dig in unscented sand to obtain small bits of chocolate. For odor discrimination, the animal's task was to learn that one scent was always correct. Which side a scent appeared on was varied over trials according to a pseudorandom Gellerman protocol. For half of the animals in each group, the cinnamon scent was correct; for half the animals, the nutmeg scent was correct. The mouse had to deduce the rule governing the correct response based on feedback on each trial (receipt or nonreceipt of the chocolate reward). Once a mouse passed the criterion for demonstrating successful learning of this discrimination, reversal learning began, and the contingencies were reversed. This procedure was repeated for a total of four reversals.

\section{Materials for odor discrimination and reversal}

Two identical circular, opaque plastic caps $(2.2 \mathrm{~cm}$ in diameter $\times 1 \mathrm{~cm}$ in depth) were aligned horizontally on a Plexiglas base $(7.5 \mathrm{~cm}$ long $\times 3 \mathrm{~cm}$ wide) (Fig. 1). The caps were $5.5 \mathrm{~cm}$ apart, center to center. The caps were attached to the base using Velcro to allow replacement after each trial so that the mouse could not use scent to mark the correct choice. One cap contained cinnamon-scented sand and one contained nutmegscented sand (the ratio of scent-to-sand was 0.3:5.0 gm). Play sand was combined with a spice (CVS-Gold brand) in a ratio of 5.0:0.03 gm sand-to-spice and thoroughly mixed. For each testing session, all caps were prepared before testing started.
A vertical barrier (5-cm-high) was positioned midway between the caps to prevent the mouse from digging in both caps on any given trial. The base was attached to a long vertical handle so that the experimenter could easily lower the apparatus into the mouse's home cage. Phe-free chocolate was used as the reward to avoid increasing the Phe levels of the PKU mice. A piece of Phe-free chocolate was buried in each of the two caps on every trial so that the mouse could not use the scent of chocolate as a cue indicating where to dig.

\section{Pretraining procedure}

All mice were weighed for $3 \mathrm{~d}$ to establish a baseline weight. Thereafter, the mice were placed on a food deprivation schedule of $90 \%$ body weight for the duration of training and testing. To minimize stress, all training and testing occurred in the animal's home cage. Only unscented sand was used during pretraining.

Pretraining phase 1. The mice were trained to dig in a cap of sand to retrieve the chocolate reward. Each trial consisted of the presentation of a single cap in the left or right position. Each training session consisted of four trials, two to each side. Three pieces of chocolate were placed in each cap on each trial in this phase training. One piece of chocolate was completely buried in the sand, the second piece was partially buried, and the third rested on top of the sand. A trial was completed successfully when the mouse retrieved all three pieces of chocolate. If the mouse failed to retrieve all three pieces of chocolate within $5 \mathrm{~min}$, the apparatus was removed and the trial was terminated. The learning criterion was four of four trials of retrieved chocolate (a total of 12 pieces of chocolate per day) for 2 consecutive days (two sessions, one per day).

Pretraining phase 2. In the second phase of training, only two pieces of chocolate were used per cap. One piece was fully buried in the sand,

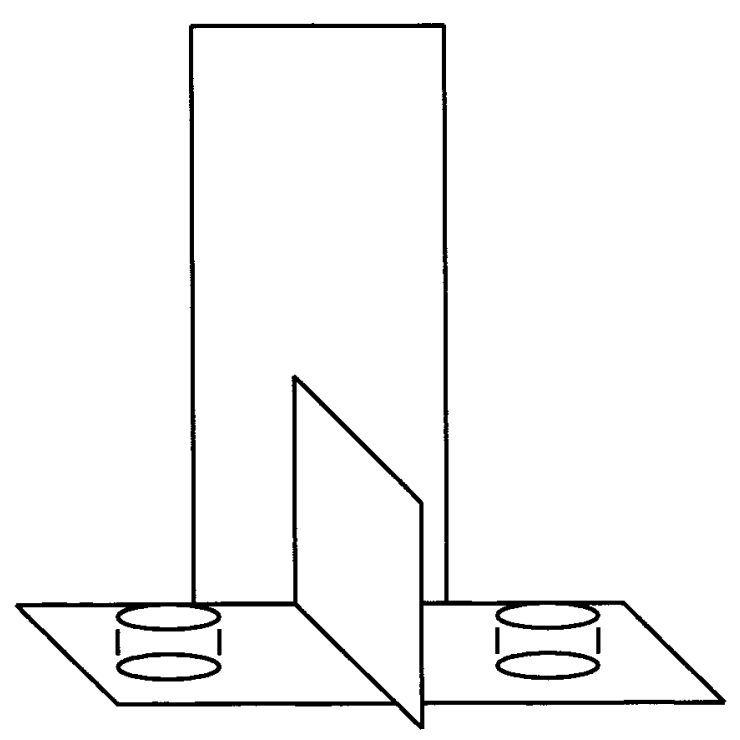

Figure 1. Line drawing of the testing apparatus used for the odor discrimination and reversal task. 
whereas the other was partially buried. A trial was completed successfully when the mouse retrieved both pieces of chocolate. If the mouse failed to retrieve both pieces of chocolate within $5 \mathrm{~min}$, the apparatus was removed and the trial was terminated. The criterion for correct performance was four of four trials of retrieved chocolate (a total of eight pieces of chocolate) in one session (four trials).

Pretraining phase 3. During the final phase of training, only one piece of chocolate was placed in the cap of sand, fully buried. Each session still consisted of four trials, one cap per trial. There were two sessions per day separated by a 5 min interval. A trial was completed successfully when the mouse retrieved the piece of chocolate. If the mouse failed to retrieve the piece of chocolate within $5 \mathrm{~min}$, the apparatus was removed and the trial was terminated. The criterion to pass this last phase of training was eight of eight trials of retrieved chocolate (a total of eight pieces of chocolate in one day) over two consecutive sessions.

\section{Odor discrimination learning procedure}

At the start of each trial, a vertical barrier was placed in the mouse's home cage. This kept the mouse away from the caps at the start of the trial. The Plexiglas cage top allowed the experimenter to slide the vertical barrier back and helped hold it in place. Next, the caps containing the nutmeg-scented and cinnamon-scented sand were lowered into the mouse's home cage. After $30 \mathrm{sec}$, the barrier was raised so that the mouse could make a choice between the two caps. A choice was scored when the mouse's nose and paws were in the sand and the mouse made a digging motion. If an incorrect choice was made, the experimenter pulled the caps out of the cage before the mouse could retrieve the chocolate. The intertrial interval was $30 \mathrm{sec}$. The intersession interval was $5 \mathrm{~min}$. A day of testing consisted of eight trials (four trials per session, two sessions per day). A mouse passed discrimination learning when 14 of 16 correct choices were made over 2 consecutive days (four sessions, two per day) within a maximum of 42 testing sessions.

\section{Odor discrimination reversal procedure}

The same testing procedure was used, except that the other previously unrewarded scent now signaled the location of the reward. A total of four reversals was administered. A mouse passed a given reversal when 14 of 16 correct choices were made over 2 consecutive days (four sessions, two per day) within a maximum of 42 testing sessions.

\section{Latent learning}

To test for latent learning, on day 1 , half the mice were allowed to explore a novel field attached to a T-maze that contained water at one location. Their learning of where the water was located is considered "latent" because they were not thirsty on day 1 . All the mice were then deprived of water for $\sim 24 \mathrm{hr}$. On day 2 , each mouse was placed in the field and maze. The question of interest was as follows: Did the mice benefit from the experience os the previous day? That is, did the mice with pre-exposure on day 1 find the water on day 2 faster then their naïve counterparts?

\section{Materials for latent learning}

The testing apparatus consisted of an open field attached to a T-maze. To minimize the likelihood of a mouse finding the water accidentally, water was always on a platform raised 2 inches above the ground, stationed at the end of the right arm of the T-maze. The left arm of the T-maze contained an identical raised platform without water. To control for scent cuing, water was placed just behind the left arm platform outside the maze. Each mouse was allowed to explore the maze alone for $5 \mathrm{~min}$ or until he/she found the water (whichever came first). If a mouse did not locate the water in $5 \mathrm{~min}$, he/she was removed from the maze. A mouse was considered to have located the water when the mouse stood on his or her hind legs and drank from the platform.

\section{Latent learning procedure}

Day 1. All mice were on full food and water. Half the mice (the pre-exposed group) were allowed to explore the T-maze that contained water at the end of the right-hand side. If a mouse did not find the water in $5 \mathrm{~min}$, he/she was removed from the maze. All the mice were then water-deprived for $24 \mathrm{hr}$.

Day 2. Each mouse was placed individually in the maze and allowed to explore. The time to locate the water was recorded. If mice in the pre-exposed group learned and remembered where the water was located, they should have found it faster than the naïve animals.

\section{Genotyping}

The group assignment of each animal (wild-type, heterozygote, or $\mathrm{Pa}$ $h^{\text {enu } 2}$ ) was independently confirmed by genotyping. DNA for genotyping each animal was obtained from 10-15 $\mu$ l of whole blood by following the manufacturer's protocol for the Isocode Stix DNA isolation procedure (Schleicher \& Schuell, Keene, NH).

Exon 7 of the $P a h$ gene, the exon mutated in $P a h^{e n u 2}$, was amplified for genotyping. Primer sequences were as follows: primer 1, 5'-ACTTGTACTGGTTTCCGCCT-3'; and primer 2, 5'-AGGTGTGTACATGGGCTTAG-3' (Genosys Biotechnologies, Woodlands, TX). The PCR mix was composed of $1 \times$ PCR buffer (containing $2 \mathrm{~mm}$ Tris-HCl, $\mathrm{pH}$ 8.0, $10 \mathrm{~mm} \mathrm{KCl,} 0.01 \mathrm{~mm}$ EDTA, $0.1 \mathrm{~mm}$ DTT, 5\% glycerol, $0.05 \%$ Tween 20 , and $0.05 \%$ Nonidet P-40), $2.5 \mathrm{~mm} \mathrm{MgCl} 2,2.5 \mu \mathrm{l}$ of DMSO, $250 \mu \mathrm{M}$ dNTPs (Boehringer Mannheim, Mannheim, Germany), 2.5 U of Taq DNA polymerase (Promega, Madison, WI), $\sim 100 \mathrm{ng}$ of template, and $1 \mu \mathrm{M}$ each primer to yield a $50 \mu \mathrm{l}$ total reaction volume. The PCR was done following the "Hot Start" PCR procedure. Cycle parameters were $95^{\circ} \mathrm{C}$ for $5 \mathrm{~min}$, followed by 30 cycles of $1 \mathrm{~min}$ at $95^{\circ} \mathrm{C}, 1 \mathrm{~min}$ at $60^{\circ} \mathrm{C}$, and $1 \mathrm{~min}$ at $72^{\circ} \mathrm{C}$. Afterward, $20 \%$ of the PCR was run on a $7.5 \%$ polyacrylamide gel until bromphenol blue reached the bottom of the gel. The gel was stained with Ethidium bromide, destained with deionized water, and visualized by UV illumination. An amplifier of $132 \mathrm{bp}$ was expected. If the amplification was successful, restriction endonuclease digestion with BsmAl or Alw26I (New England Biolabs, Beverly, MA) was performed and incubated at 55 or $37^{\circ} \mathrm{C}$, respectively, overnight. The digestion products were run on a polyacrylamide gel as described above. The diagnostic fragments were as follows: wild-type, 82 and $50 \mathrm{bp}$; heterozygote, $82,50,48$, and $34 \mathrm{bp}$; and homozygote, 50,48 , and $34 \mathrm{bp}$.

\section{RESULTS}

\section{Results for odor discrimination and reversal}

There were no age differences on any dependent measure. Therefore, age was not entered into any of the analyses reported here. $P a h^{\text {enu } 2}$ (PKU) mice were not impaired on acquisition of odor discrimination. Female mice, however, took significantly longer to acquire the odor discrimination than male mice $\left(F_{(2,39)}=5.28\right.$; $p<0.03)$. There were no significant sex by group interactions and no sex differences in performance on reversals. Pah ${ }^{\text {enu2 }}$ (PKU) mice were impaired on reversals 2,3 , and 4 of the odor reversal task, even controlling for sex. Thus, for example, on the number of days to reach criterion, there was a main effect for group on reversal $2\left(F_{(2,37)}=4.70 ; p<0.05\right)$, reversal $3\left(F_{(2,34)}=4.14 ; p<\right.$ $0.05)$, and reversal $4\left(F_{(2,27)}=3.66 ; p<0.05\right)$ (Fig. $\left.2 A\right)$. Using the Tukey test for post hoc comparisons between each of the groups, $P_{a h}{ }^{\text {enu }}$ (PKU) mice took significantly longer to reach criterion on reversal 2 than wild-type $(4.38 ; p<0.03)$ and heterozygous $(6.15 ; p<0.01)$ mice, whereas there were no significant differences between the performance of wild-type and heterozygous mice. Pah ${ }^{\text {enu } 2}$ (PKU) mice also took significantly longer than wild-type mice to reach criterion on reversal $3(6.48 ; p<0.03)$ and reversal $4(6.29 ; p<0.03)$. The heterozygous mice performed intermediate between these two groups and so did not differ significantly from either Pah ${ }^{\text {enu } 2}$ (PKU) or wild-type mice in the number of days to reach criterion on reversals 3 or 4 .

Whereas the Pahenu2 (PKU) animals performed significantly worse on reversals $2-4$, this was not true for reversal 1 . To investigate whether our failure to find an impairment in the $\mathrm{Pah}^{\text {enu2 }}$ (PKU) mice on reversal 1 was attributable not so much to the good performance of that group but to the poor performance of a few of the control animals, we re-ran the analysis omitting the two worst performing animals from the wild-type and heterozygous groups. Doing that, the main effect for group in the number of days to reach criterion on reversal 1 was significant $\left(F_{(2,32)}=\right.$ 3.37; $p<0.05$ ), and Tukey post hoc comparisons showed the PKU 
A

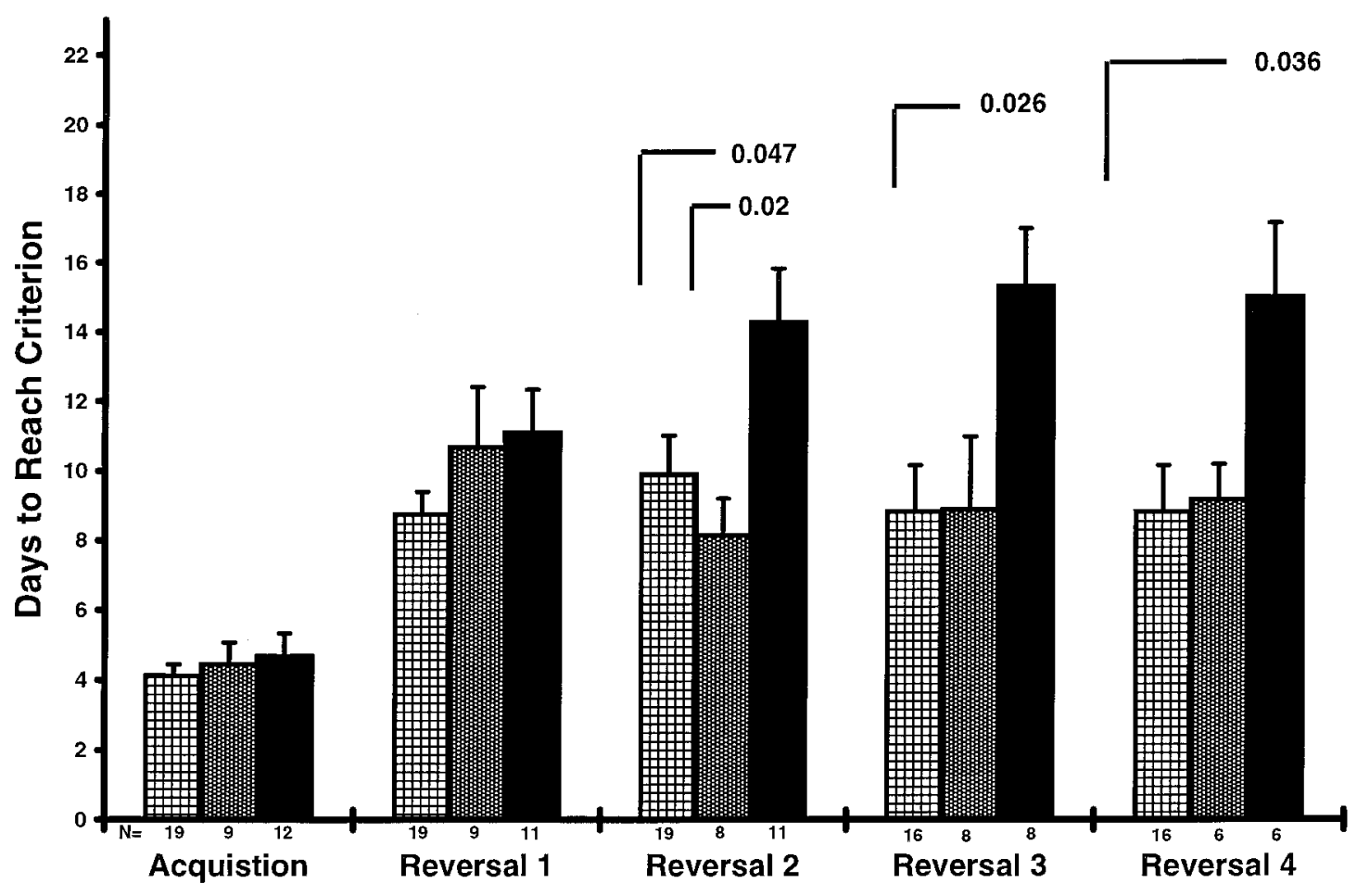

(Odor Discrimination)

B

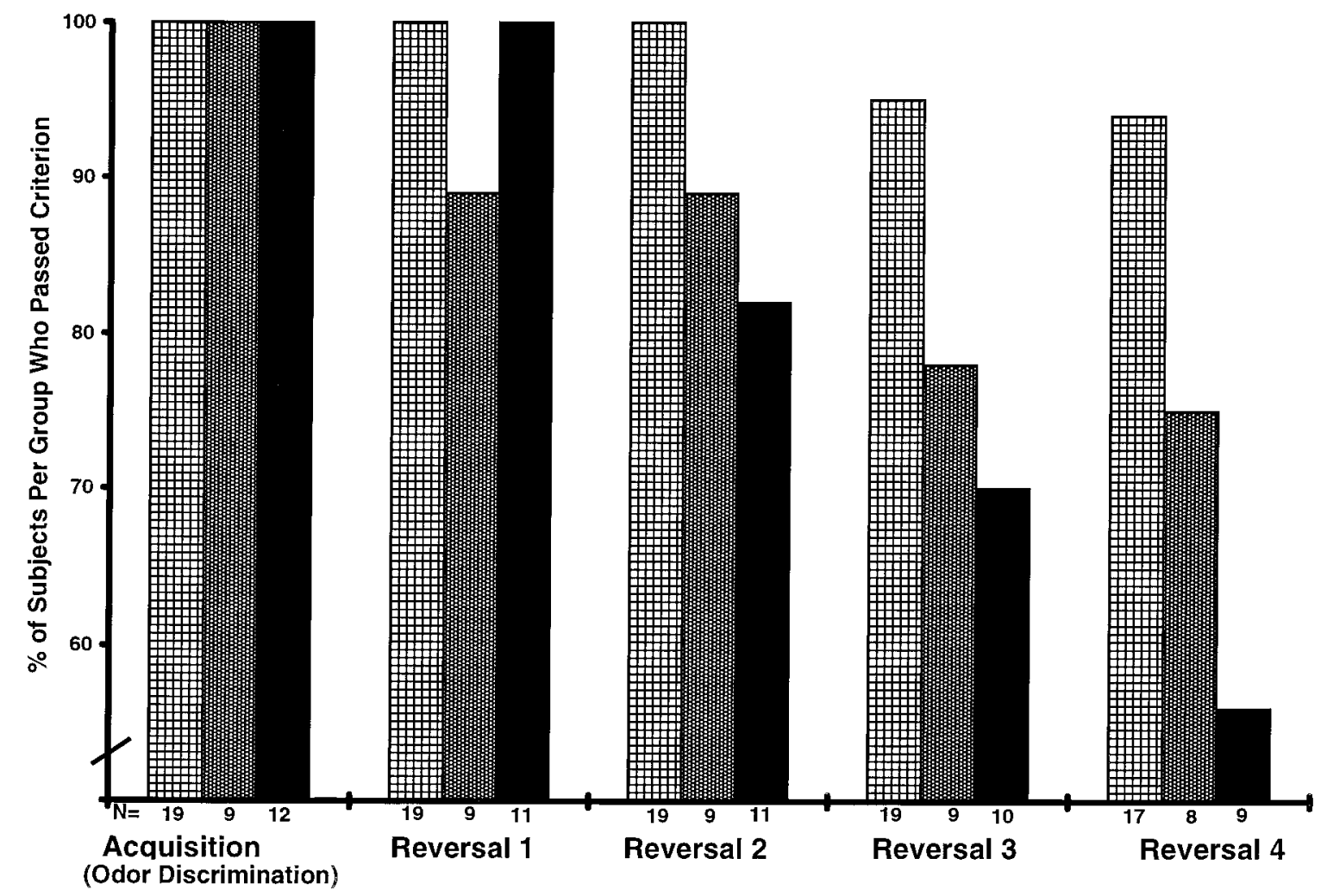

Figure 2. Performance of the two control groups [wild-type and heterozygotes (wild-type crossed with Pah ${ }^{\text {enu } 2}$ [PKU])] and the PKU model animals $\left(P h^{\text {enu } 2}\right)$ on odor discrimination and reversal. The PKU model animals acquired the odor discrimination normally and performed well on the first reversal. However, as can be seen by the number of days to reach criterion $(A)$ and by the percentage of subjects per group to pass criterion $(B)$, the PKU model animals were performing worse than the control groups by the last reversals. Criterion was less than or equal to two errors over 2 consecutive

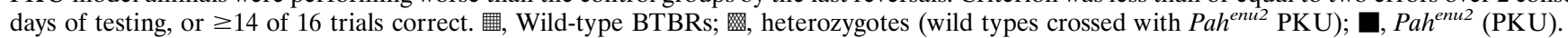




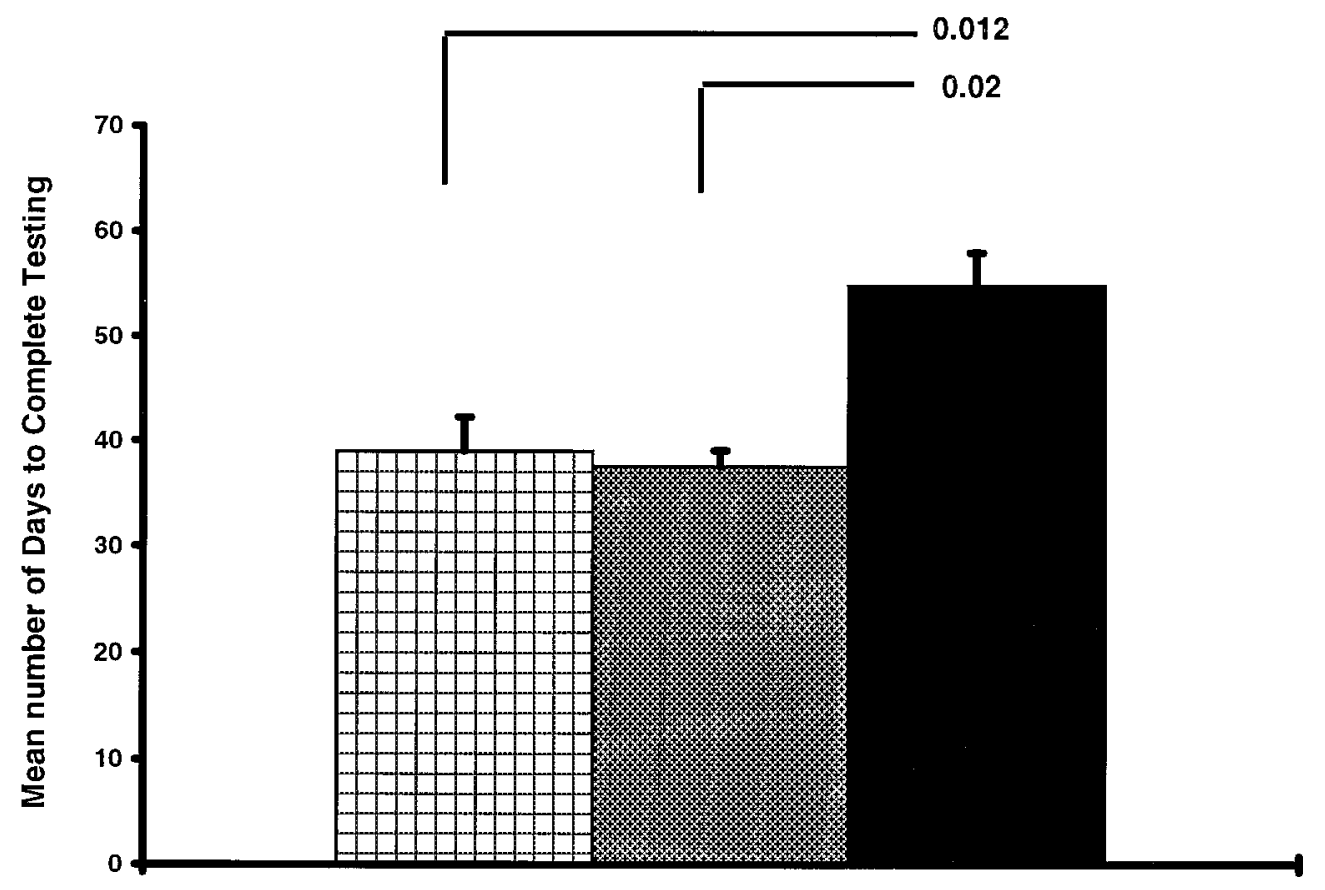

\begin{abstract}
Figure 3. Total number of days to complete testing on odor discrimination and reversal. The PKU model animals took significantly longer to complete testing. No significant difference emerged between the heterozygous and wild-type groups. 湡, Wild-type BTBRs; 网, heterozygotes (wild types crossed with $\mathrm{Pa}$ $h^{\text {enu2 }}$ PKU); $\mathbf{Q}, \mathrm{Pah}^{\text {enu2 }}$ (PKU).
\end{abstract}

model animals to be performing significantly worse than the wild-type animals $(2.86 ; p<0.03)$ and tending to perform worse than the heterozygous animals $(2.81 ; p<0.07)$.

$\mathrm{Pah}^{\text {enu } 2}$ (PKU) mice also took significantly longer to finish four reversals if one considers all of the testing from acquisition through reversal 4 (Fig. 3). Thus, there was a main effect for group on the total number of days to complete testing $\left(F_{(2,26)}=\right.$ 5.72; $p<0.01)$. Using the Tukey test for post hoc comparisons, Pah $^{\text {enu } 2}$ (PKU) mice took significantly longer than wild-type (15.83; $p<0.01)$ and heterozygous $(17.33 ; p<0.005)$ mice in the total number to complete testing. No significant difference emerged between the heterozygous and wild-type groups.

The percentage of $\mathrm{Pah}^{\text {enu } 2}$ (PKU) mice who passed reversals 3 and 4 was much lower than the percentages in the two other groups (percent passing Reversal 3, Pahenu $(\mathrm{PKU}), 70 \%$; heterozygotes, 78\%; wild-type, 95\%; Reversal 4, Pah $^{\text {enu } 2}$ (PKU), 56\%; heterozygotes, 75\%; wild-type, $88 \%$ ) (Fig. 2B). However, so few $\mathrm{Pah}^{\text {enu }}$ (PKU) animals passed earlier reversals that the number of Pah ${ }^{\text {enu } 2}$ (PKU) mice tested on reversals 3 and 4 was too small to yield statistically significant differences when compared with the other groups. On this measure, as on all other measures, there was no significant difference between the performance of wild-type and heterozygous mice.

\section{Evidence that the group effects were not caused by differences in motivation, activity, or olfactory ability}

All animals were kept at $85-90 \%$ body weight to ensure they would be hungry. No animals gave any indication of being unmotivated. The animals in all groups chose quickly on all trials. The mean latency to begin digging in the sand was identical for the $P_{a h}{ }^{\text {enu2 }}$ (PKU) mice and the wild-type mice $(13.20 \mathrm{sec})$. No group seemed more active or more lethargic than any other.

The fact that mice in all groups performed well and comparably at the initial discrimination and initial reversal strongly suggests that the mice in all groups were able to detect the scents and that the groups did not differ in this ability. Further evidence that the mice could smell the scents is the typical behavior of most animals in all three groups once they began performing well on the initial discrimination; Mice in all groups would go over to a container of sand and sniff it before committing to that choice. The olfactory ability of the mice in all groups was superior to our own, because their performance during our pilot work indicated that they could smell chocolate buried in the sand, although we could not. When we hid the chocolate in only one cap of sand on each trial and randomly varied the side of the sand with the buried chocolate, the animals in all three groups chose the correct side better than would be predicted by chance. For that reason, in the work reported here, we adopted the procedure of burying chocolate in both caps of sand on each trial.

\section{Results for latent learning}

There were no age or sex differences in performance on latent learning. Therefore, neither age nor gender were entered in the analyses of group differences on this task.

$P_{a h}{ }^{\text {enu2 }}$ (PKU) mice failed to show latent learning (Fig. 4). That is, $P a h^{\text {enu } 2}$ (PKU) mice pre-exposed to the field and maze on day 1 did not find the water significantly faster on day 2 when compared with the naïve PKU animals $\left(F_{(1,17)}=1.30\right.$; NS). On the other hand, wild-type and heterozygote animals pre-exposed to the field and maze on day 1 showed a significant benefit from that pre-exposure (they had learned and remembered where the water was located) compared with the naïve wild-type or naïve heterozygote animals (wild-type animals, $F_{(1,29)}=6.12 ; p<0.03$; heterozygous animals, $\left.F_{(1,30)}=5.33 ; p<0.03\right)$. This is not an artifact of any difference in baseline or in reaction to a novel environment (Fig. 5). There were no significant differences among the groups in the percentage of animals not previously exposed to the maze who found the water on day 1 or day 2 . There were no significant differences among the groups in time needed to locate the water by animals not previously exposed to the maze $\left(F_{(2,39)}=0.15\right.$; NS). There were no differences between the performance of wild-type and heterozygous mice on any measure or comparison. 
Figure 4. Performance of the two control groups [wild-type and heterozygotes (wild-type crossed with $P a h^{\text {enu } 2}$ [PKU])] and the PKU model animals $\left(\mathrm{Pah}^{\text {enu }}\right)$ on latent learning. Although no animals had been water-deprived on day 1 , animals in the two control groups (heterozygotes and wild-type) who were exposed to the open field and maze on day 1 remembered where the water was located in the maze and were able to use that to find the water faster on day 2 than naïve animals. In contrast, PKU model animals pre-exposed to the field and maze on day 1 were not able to find the water significantly faster than naïve PKU model animals. The few animals in the pre-exposed group who failed to find the water on day 1 are not included in this graph. 畻, Pre-exposed group; 㘣, naïve group.

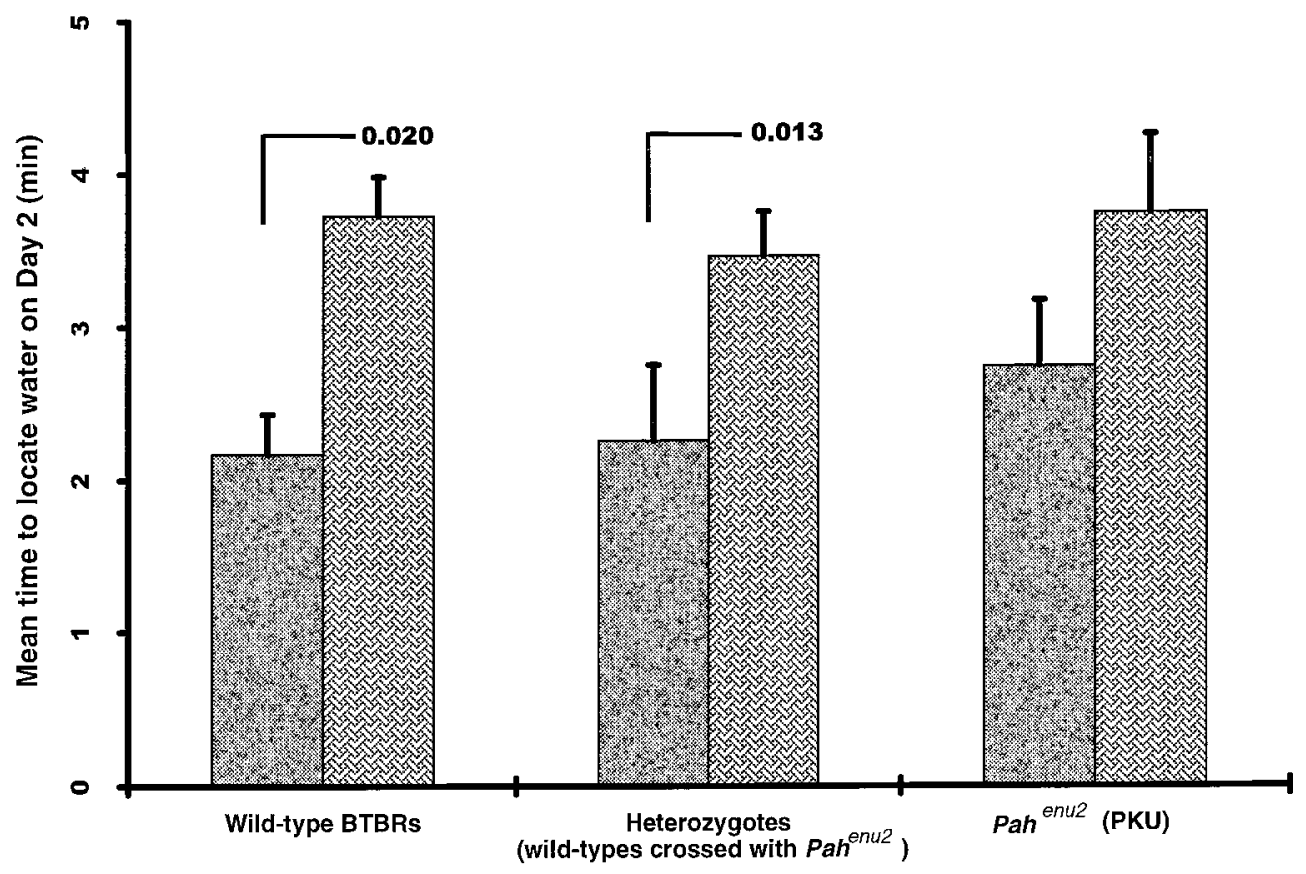

(The few animals in the pre-exposed group who failed to find the water on Day 1 are not included in this graph.)

\section{DISCUSSION}

\section{Discussion of procedural issues}

For the odor discrimination and reversal procedure, we built on and modified a procedure originally developed for rats by Bunsey and Eichenbaum (1996) and subsequently modified by Libbey et al. (1996) and Berger-Sweeney et al. (1998) for mice. The older procedures had certain problems, which we have corrected. (1) Some mice adopt the strategy of simply going to the choice that happens to be closest to them when the testing apparatus is lowered into their cage. To prevent this maladaptive strategy, we placed a clear Plexiglas barrier in front of where the two choices were to be lowered. This forced the mouse back. The apparatus was lowered, and then the barrier was removed. This significantly cut down on the time needed to train the mice compared with our preliminary results using the Berger-Sweeney et al. (1998) procedure. (2) Before conducting the work reported here, we conducted control tests (with two choices of sand with the same scent; the chocolate reward randomly placed in the right or left choice). The control tests indicated that the mice were able to smell the chocolate, although it was buried deep in the sand and the sand was scented. Therefore, for testing, we hid chocolate at the bottom of both sand caps. If the mouse chose the wrong cap, we allowed the mouse to dig a bit and then removed the two caps. Thus, it was impossible to use the scent of the reward to help guide a response.

(3) In our preliminary work with this procedure, when a mouse made a wrong choice, the mouse so quickly scurried over to the correct choice that it had been difficult to prevent the animal from being rewarded, although the animal's first choice had been incorrect. To prevent the animal from retrieving the reward from the incorrect cap, we erected a clear Plexiglas wall between the two choices. The mice responded to this as we had hoped; they smelled at each choice and only then chose one of the caps of sand. (4) Before we modified the procedure, mice were able to cheat by marking the cap of sand with their saliva. To prevent this strategy, we substituted a new cap with sand of the same scent on the next trial. That is, we never re-used the same cap or sand in a testing session once a mouse had gone to it. (5) Finally, BergerSweeney et al. (1998) report that the discrimination on which they trained mice was sand scented with cinnamon versus sand scented with curry. In our preliminary work, we found that our mice preferred cinnamon over curry, and so initial acquisition and reversal were easier when cinnamon was the $\mathrm{S}+$ (rewarded scent) and harder when curry was the correct choice. This added noise to the data. After some experimentation, we found that the scents of cinnamon and nutmeg were approximately evenly matched for our mice, and thus we used those in our testing.

\section{Conclusions}

PKU causes cognitive deficits in humans if it is untreated. We have found the first evidence that the genetic mouse model of PKU exhibits cognitive impairments. PKU mice were impaired on reversals 2, 3, and 4 of the odor reversal task. PKU mice took longer to learn each successive reversal. They showed no evidence of having benefited from previous experience with the task.

PKU mice were also impaired in latent learning. PKU mice found the water on day 1 , as did the wild-type and heterozygote animals pre-exposed on day 1 . However, whereas wild-type and heterozygous pre-exposed mice showed evidence that they remembered where the water was located and could use that to find the water faster on day 2, the pre-exposed PKU animals did not show a similar savings on day 2 . Wild-type and heterozygous mice who were exposed to the location of the water on day 1 found it significantly faster on day 2 than naïve animals. PKU mice, however, who found the water on day 1 did not find the water significantly faster on day 2 than naïve PKU animals. They showed no significant benefit from their pre-exposure on the preceding day. 
A

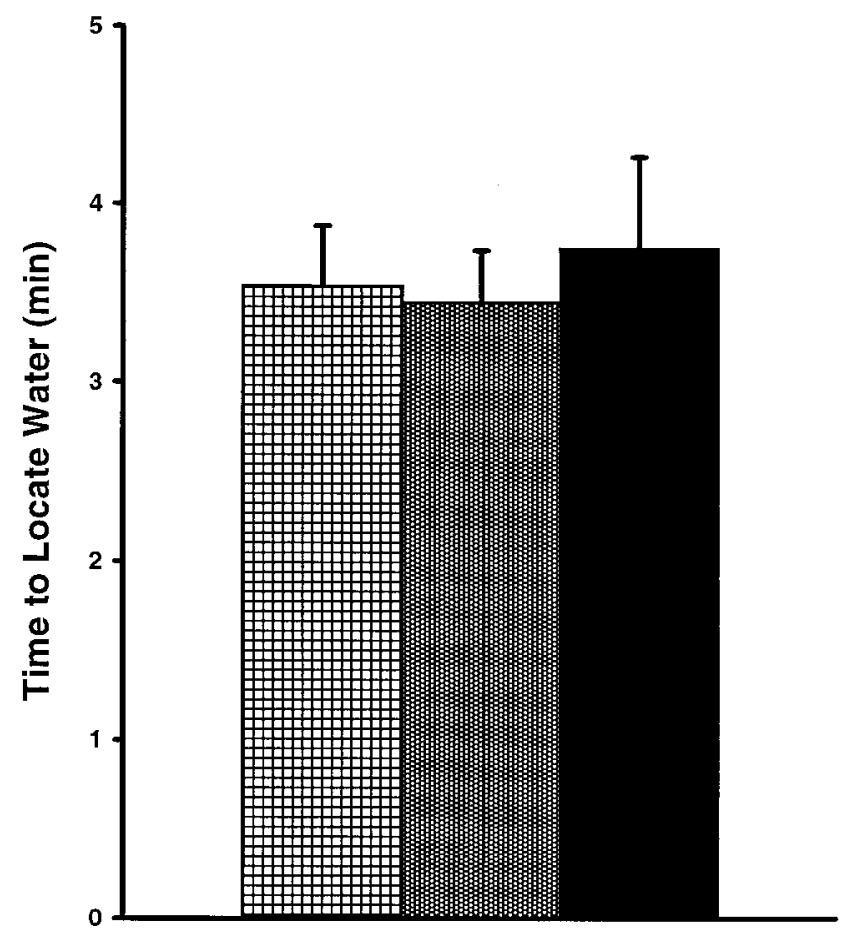

B

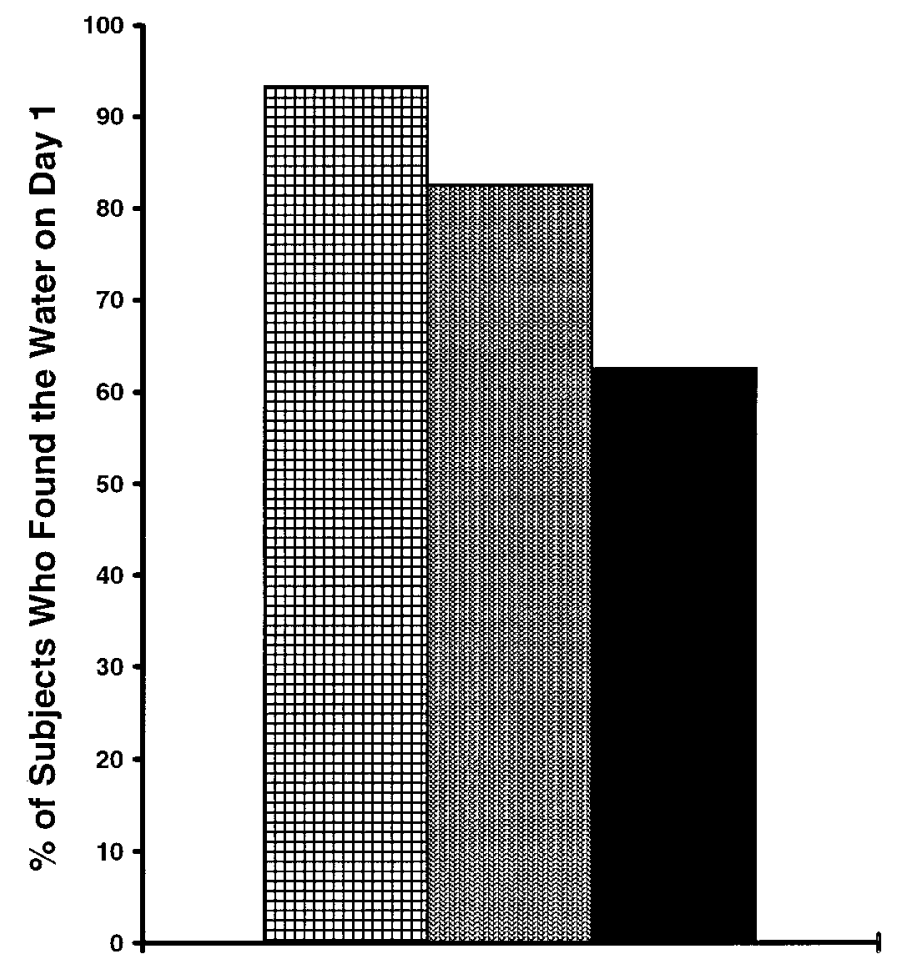

Figure 5. Performance of naïve animals on their first exposure to the latent learning environment. There were no significant differences between the

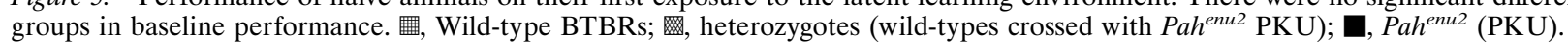

These cognitive deficits, although significant, were not incapacitating and do not seem to constitute profound mental retardation. For example, the PKU model animals were able to learn odor discrimination (although they were significantly impaired on reversals 2-4), and most were able to find water in the open field plus T-maze (although they showed little or no benefit from the exposure of the previous days). It may be difficult to obtain evidence of major cognitive deficits in mice because at least a few "normal" mice do not appear to be very bright, at least not on the laboratory tasks correctly in use by behavioral neuroscientists. Thus, when the two worst performing animals from our wild-type and heterozygous control groups were omitted from the analysis, it could be clearly seen that the PKU model mice were performing significantly worse than most of the mice in the comparison groups. Against a background of relatively poor performance in at least some normal mice, it may be difficult to find dramatically worse performance in "retarded" mice.

Investigators working with a genetic mouse model of another disorder that causes mental retardation, Fragile X, have obtained results of cognitive deficits quite comparable with what we have found here (Dutch-Belgian Fragile X Consortium, 1994). Using the Morris water maze, they found that their mutant animals learned to find the hidden platform at the same rate as their normal littermates and showed comparable spatial memory of the location of the platform. However, when the position of the platform was changed on reversal trials, the Fragile X model mice were significantly impaired. Thus, in these two genetic mouse models of conditions that cause mental retardation in humans, PKU and Fragile X, the mutant mice showed normal learning of the initial condition but were impaired on reversals. Persons with mental retardation similarly can learn simple discriminations but show impairments when discriminations are reversed (House and Zeaman, 1962).

In addition to their cognitive deficits, the PKU model animals were extremely reactive to stress. For that reason, we went to considerable lengths to minimize stress in our procedures. Some of the PKU model animals never acclimated to human touch, even when we introduced this daily beginning during the first week of life. Therefore, the mice used in this study were handled as little as possible and were not touched during testing. We tested them on odor discrimination and reversal in their home cages and transported them to the latent learning apparatus in a small Plexiglas transport box that they could enter and leave without human touch. Many investigators use escape from aversive conditions (e.g., water mazes) as the reward when testing mice. In preliminary work, we found that our PKU mice were so stressed by being placed in water that they froze rather than trying to escape. We have used food and water rewards in the work reported here.

\section{REFERENCES}

Berger-Sweeney J, Libbey M, Arters J, Junagadhwalla M, Hohmann CF (1998) Neonatal monoaminergic depletion in mice (Mus musculus) improves performance of a novel odor discrimination task. Behav Neurosci 112:1318-1326.

Bunsey M, Eichenbaum H (1996) Conservation of hippocampal memory function in rats and humans. Nature 6562:255-257.

Cowie VA (1971) Neurological and psychiatric aspects of phenylketonuria. Phenylketonuria and some other inborn errors of amino acid metabolism. (Bickel H, Hudson FP, Woolf LI, eds). Stuttgart, Germany: Verlag. 
DiLella AG, Marvit J, Lidsky AS, Güttler F, Woo SLC (1986) Tight linkage between a splicing mutation and a specific DNA haplotype in phenylketonuria. Nature 322:799-803.

Dutch-Belgian Fragile X Consortium (1994) Fmr1 knockout mice: a model to study fragile $X$ mental retardation. Cell 78:23-33.

House B, Zeaman D (1962) Reversal and non-reversal shifts in discrimination learning in retardates. J Exp Psychol 63:444-451.

Hsia DY (1966) Inborn errors of metabolism. Chicago: Year Book Medical.

Koch R, Azen C, Friedman EG, Williamson EL (1982) Preliminary report on the effects of diet discontinuation in PKU. Pediatrics 100:870-875.

Libbey M, Hohmann C, Berger-Sweeney J (1996) Neonatal 5,7-DHT lesions alter performance of passive avoidance and a novel odor discrimination task in adult mice. Soc Neurosci Abstr 22:681.
Lidsky AS, Law ML, Morse HG, Kao FT, Woo SLC (1985) Regional mapping of the human phenylalanine hydroxylase gene and the PKU locus on chromosome 12. Proc Natl Acad Sci USA 82:6221-6225.

McDonald JD, Bode VC, Dove WF, Shedlovsky A (1990) Pah ${ }^{\text {hph-5: a }}$ mouse mutant deficient in phenylalanine hydroxylase. Proc Natl Acad Sci USA 87:1965-1967.

Shedlovsky A, McDonald JD, Symula D, Dove WF (1993) Mouse models of human phenylketonuria. Genetics 134:1205-1210.

Tourian AY, Sidbury JB (1978) Phenylketonuria. The metabolic basis of inherited disease. (Stanbury, JD, Wyngaarden JB, Fredrickson D, eds), pp 240-255. New York: McGraw Hill.

Woo SLC, Lidsky AS, Güttler F, Chandra T, Robson, KJH (1983) Cloned human phenylalanine hydroxylase gene allows prenatal diagnosis and carrier detection of classical phenylketonuria. Nature 306:151-155. 